\title{
Human Prediction of Computer Generated Value Based on Statistical Experimental Approach Intuition in Human-Computer Interaction Research
}

\author{
Radim Brixí \\ Department of System Analysis at Faculty of Informatics and Statistics \\ University of Economics, Prague \\ Prague, Czech Republic (EU) \\ e-mail: radim.brixi@vse.cz
}

\begin{abstract}
Paper describes statistically significant anomaly in statistical results from test scenarios of human prediction success rate of right button generated by computer. The prediction success rate seems to be slightly higher than the theoretical probability. Paper presents seven test runs with different number of steps where all tests proved higher human performance than probable. The paper also presents prediction learning application approach even though its effectiveness was not yet measured. Test was performed by a very spiritual person.
\end{abstract}

Keywords-- prediction, intuition, computer generated values

\section{INTRODUCTION}

Spiritual people come across phenomenon like insight or prediction or intuition. We focus on ability and possibility to predict generated value even though no information about computer generated value is obtained by the user through physical senses. We test whether the cognitive process can lead to better statistical results than the probability of the test case supporting the testing with belief of success. Experiment in [1] describes the collective consciousness possible impact into the physical world changes influencing statistically collected data. Also Roger Nelson's Global Consciousness Project reflects statistically significant changes in randomly generated data when global consciousness is affected by global event [2]. These and similar research results very much influence interpretation and understanding of human-world and human-computer interaction and human's influence of the consciousness on outer reality. Quantum entanglement of all particles implicates in thoughts about connection between consciousness and reality. The anomaly we face in this paper is that no known cognitive process is present even though the statistical result of the performed tests tend to indicate that the human prediction performance seem to be slightly better than the theoretical probability value in all presented test runs that would be performed by computer statistically-based approach of guessing unknown value.

\section{RESEARCH METHODS AND PROCESS}

Experimental research is based on simple application which is used for prediction testing (and also possibly for teaching). User selects number of buttons to perform prediction experiments among which the user is supposed to choose one. The application calculates random button that the computer user is supposed to guess. The random button is calculated by the computer already when the buttons are shown even though they all look the same and no visual information is available. Buttons are labeled 1-3 only for purpose of identification. The computer performs random calculation which button is supposed to be pressed by the user before the user is supposed to guess the right button. The calculation and the randomly chosen button are only in computer memory and none of this information is displayed on the monitor for the user being tested until the user makes his choice. (Future tests should measure differences in results when user clicks the button before the calculation process of random button is pressed or while randomizing process dynamically changes the random button several hundred times per second during the testing time.)

When user guesses and presses a button that the user things the computer randomly chose, one of two possibilities may occur. Either the user guessed right and chose correct button, so the attempt is logged as correct or the user guessed wrong button so the originally computer generated is displayed in green and the user must click the green button to simulate correction and learning process of guessing the right button and the attempt is logged as wrong. Every step result is logged as three numbered values: button number pressed, 1 if correctly guessed or nothing and finally number generated by computer. The cognitive process can use only visual information from the application that carries no information about what computer generated (and other irrelevant information that come into the cognitive process of the user). The test was performed with strong belief that the user can influence the calculated success rate in such a way that the ratio of successfully guessed buttons divided by total number of guesses is going to be significantly greater than the theoretical probability.

To simulate teaching process (although with no clue how to learn) prediction learning is implemented in the application in such a way that every time the user guesses wrong button, he must correct himself by clicking suddenly on green coloured button. The button is greened by the computer so the user learns which button he was supposed to click on even though he clicked the wrong button. This effect supports the belief and confidence and reflects principles of learning without knowing the learning process. Differences in application visual state are shown on Fig. 1. and Fig. 2. 


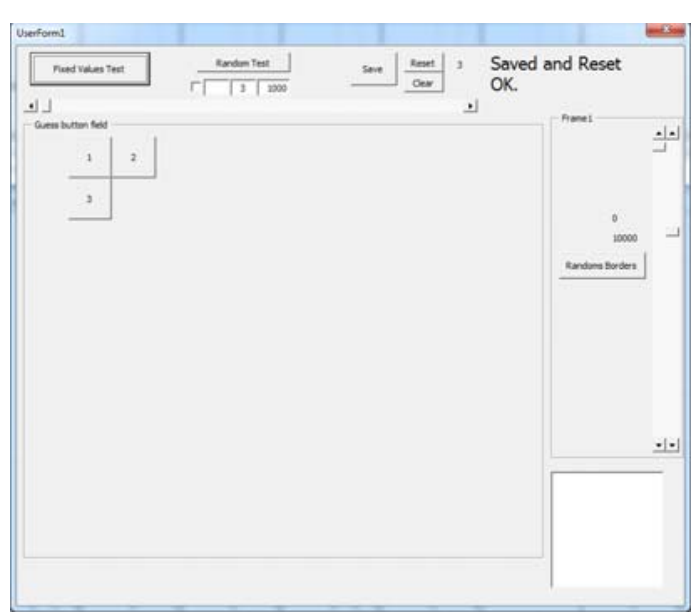

Figure 1. Application in the mode ready for guessing.

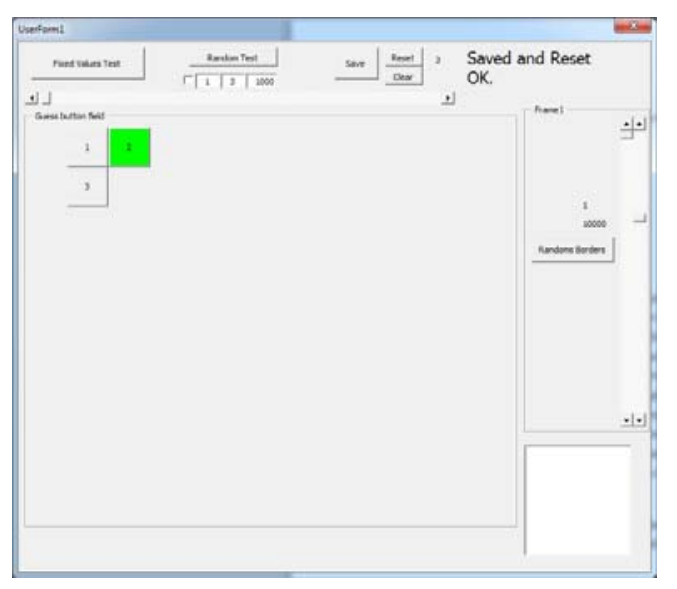

Figure 2. Application in the mode ready for guessing.

In case of three buttons the probability is $1 / 3$ that randomly chosen button by computer is guessed right based on randomly selected button from all of the three displayed buttons.

Used statistical method used for experimental research is based on hypothesis that the test application user performance is higher than the theoretical probability level.

We assume that the distribution is approximately normalized normal.

Hypotheses are $\mathrm{H}_{0}: \pi=1 / 3$ for three buttons runs and $\mathrm{H}_{0}$ : $\pi=1 / 6$ for six buttons run with $H_{1}: \pi>1 / 3$ for three buttons runs and $\mathrm{H}_{1}: \pi>1 / 6$ for six buttons run.

The test criteria used are described in following formula:

$$
u=\frac{p-\pi_{0}}{\sqrt{\frac{\pi_{0}\left(1-\pi_{0}\right)}{n}}}
$$

Significance level $\mathrm{W}_{0.05}\left\{u ; u \geq u_{0.95}\right\} ; u_{0.95}=1.645$.

The following Table 1 describes detailed test results.

\section{CONCLUSION}

Because the test was performed by very spiritual type of person, we should focus in further research how spiritual people observe things, how cognitive processes vary depending on level of spirituality of different kinds and what does it implicate for computer technology and its applications. The anomaly can be explained by luck, but the measured probability seems to be too low for that even though possible. The longest test run consisted through 2564 steps of the test and this test proves on large amount of data the hypothesis.

Test results should be a new guideline for creating robotic cognitive parts of robot architecture types like in [3]. We understand the effect of consciousness on outer world yet too little, this paper measures potential process that exists beyond known cognitive research areas because the observation of information needed for decision cannot be observed through information displayed at the screen. The test results seem to tend to discussion that inner observation seems to be involved somehow in the cognitive process resulting in better decision making. Understanding this topic may open new gates to new technologies and machine architectures. This has also serious impact on security issues because the probability that one guesses for example password seem to be possibly slightly higher for the human user compared to bot in the same number of attempts to guess it.

Knowledge of humans seem to be based on more than visible aspects and therefore designing computer processes, artificial intelligence, machine learning mechanisms or applications based on similarities how humans work should count with results from this paper, that humans use also noticeable portion of intuition.

\section{REFERENCES}

[1] Hagelin, John S, Rainforth, Maxwell V, Orme-Johnson, David W, Cavanaugh, KENNETH L, \& et al. (1999). Effects of group practice of the Transcendental Meditation program on preventing violent crime in Washington, D.C.: Results of the National Demonstration Project, June-July 1993. Social Indicators Research, 47(2), 153-201.

[2] Global Consciousness Project [online]. 2012 [cit. 2012-1125]. www: http://noosphere.princeton.edu/

[3] BRIXÍ, Radim, BRIXÍ, Sylvie. The Association Reactor in a Human-like Robot Architecture Concept: An Interdisciplinary Approach. Jindřichův Hradec 09.09.2009 - 11.09.2009. In: IDIMT-2009 System and Humans - A Complex Relationship. Linz: Trauner Verlag universitat, 2009, s. 297-308. ISBN 978-3-85499624-8. 
Table 1. Seven tests of human prediction of randomly generated button (First test was run with six buttons, other with three buttons)

\begin{tabular}{|c|c|c|c|c|c|c|c|}
\hline Test run number & 1 & 2 & 3 & 4 & 5 & 6 & 7 \\
\hline Number of steps in the test run & 1000 & 83 & 100 & 142 & 389 & 234 & 2564 \\
\hline Successfully guessed & 193 & 38 & 45 & 57 & 148 & 91 & 899 \\
\hline Number of buttons & 6 & 3 & 3 & 3 & 3 & 3 & 3 \\
\hline $\begin{array}{c}\text { Test criteria value u with } \\
\text { critical value 1,645 }\end{array}$ & 2.234 & 2.406 & 2.475 & 1.721 & 1.972 & 1.803 & 1.857 \\
\hline $\begin{array}{c}\text { Measured average score } \\
\text { Theoretical probability }\end{array}$ & 0.193 & 0.4578313 & 0.45 & 0.4014085 & 0.3804627 & 0.3888889 & 0.350624 \\
\hline
\end{tabular}

